\title{
Peripheral Artery Tonometry Reveals Impaired Endothelial Function before Percutaneous Coronary Intervention in Patients with Periprocedural Myocardial Injury
}

\author{
Zhangwei Chen $\left(\mathbb{D},{ }^{1}\right.$ You Zhou $\left(\mathbb{D},{ }^{2}\right.$ Jiasheng Yin $\left(\mathbb{D},{ }^{1}\right.$ Qinglai Gao, ${ }^{3}$ Ao Chen, ${ }^{1}$ Yan Xia $\left(\mathbb{D},{ }^{1}\right.$ \\ Danbo Lu $\mathbb{D}^{1}$, Dong Huang, ${ }^{1}$ Juying Qian $\mathbb{D}^{1}{ }^{1}$ and Junbo Ge $\mathbb{D}^{1}$ \\ ${ }^{1}$ Department of Cardiology, Zhongshan Hospital, Fudan University, Shanghai Institute of Cardiovascular Diseases, \\ National Clinical Research Center for Interventional Medicine, Shanghai 200032, China \\ ${ }^{2}$ Department of Cardiology, Zhongshan Hospital, Fudan University, Shanghai Geriatric Medical Center, Shanghai \\ Institute of Cardiovascular Diseases, National Clinical Research Center for Interventional Medicine, Shanghai 200032, China \\ ${ }^{3}$ Department of Cardiology, People's Hospital of Wulian, Rizhao, Shangdong Province 262300, China
}

Correspondence should be addressed to Juying Qian; qian.juying@zs-hospital.sh.cn and Junbo Ge; jbge@zs-hospital.sh.cn

Received 13 February 2021; Accepted 5 July 2021; Published 15 July 2021

Academic Editor: Martin J. Swaans

Copyright (c) 2021 Zhangwei Chen et al. This is an open access article distributed under the Creative Commons Attribution License, which permits unrestricted use, distribution, and reproduction in any medium, provided the original work is properly cited.

\begin{abstract}
Background. Periprocedural myocardial injury (PMI) is a most common complication of percutaneous coronary intervention (PCI). Microembolization and inflammation underlying PMI could lead to coronary microvascular dysfunction (CMD) and vice versa. Reactive hyperemia index (RHI) assessed by peripheral artery tonometry (PAT) has been considered as a noninvasive method to assess endothelial function and CMD, which could be useful to predict PMI. Methods. 268 patients suspected with stable coronary artery disease (CAD) and scheduled for elective coronary angiography were enrolled. RHI was measured by using the Endo-PAT2000 ${ }^{\mathrm{rm}}$ device before angiography. The association among RHI, PMI, and cardiovascular events was further assessed. Results. In this cohort, 189 patients (70.5\%) were diagnosed with CAD and 119 patients (44.4\%) underwent drug-eluting stent (DES) implantation. Compared with patients without CAD, CAD patients had lower RHI $(1.88 \pm 0.55$ vs. $2.02 \pm 0.58$, $P<0.05)$. Patients with PMI had a lower RHI before angiography $(1.75 \pm 0.37$ vs. $1.95 \pm 0.50, P<0.05)$. Receiver operating characteristic curve analysis of RHI revealed an area under the curve (AUC) of 0.61 , with a sensitivity of $62.7 \%$ and specificity of $50.0 \%$ to predict PMI. Moreover, we found that CAD patients with $\mathrm{RHI} \leq 1.81$ had a higher incidence of composite cardiac events after stenting (adjusted hazard ratio (HR) 3.31, 95\% confidence interval (CI) 1.07-10.22, $P<0.05$ ). Conclusions. RHI assessment through PAT could be a promising method to predict PMI before the procedure. RHI is associated with increased risk of long-term adverse cardiac events after DES implantation.
\end{abstract}

\section{Introduction}

PCI has been a standard approach to achieve revascularization of obstructive coronary CAD. The improvement of techniques and equipment dramatically decreases the incidence of severe complications after PCI, such as stent thrombosis, perforation, and death [1]. However, PMI emerges as a frequent complication of PCI with an incidence of 5-70\% depending on the diagnostic criteria [2-4]. Previous studies imply an association between PMI and adverse cardiovascular events [4-6]. The diagnosis of PMI depends on the values of cardiac troponin (cTn) or creatine kinaseMB (CK-MB) after the procedure. Yet, no ideal method has been found to predict or prevent PMI effectively. Herein, we intend to find out risk factors or examinations that could identify patients at high risk of PMI before the procedure, so that we can adopt preventive measures to reduce the damage of PMI.

The most common mechanisms of PMI could be classified into two types: one is due to side branch occlusion 
proximal to the target lesion of PCI, and the other is due to microvascular dysfunction distal of the treated lesion [7]. The distal type is believed to be the dominant type of PMI, which is closely related with embolization of the arteriole, vascular dysregulation, and microcirculatory dysfunction [8-11]. CMD is a crucial pathophysiologic damage caused by PMI, which, in turn, could be an important risk factor that predisposes patients to PMI. PAT, which detects the reactive hyperemia-induced changes in digital peripheral pulse amplitude to evaluate peripheral endothelial function [12], was not only closely related with cardiovascular risk factors [13] but also considered as a valuable noninvasive way to evaluate coronary microvascular function $[14,15]$. Hence, PAT could be a useful method to identify patients at high risk of PMI before the procedure.

Impaired endothelial function detected by PAT was associated with higher adverse cardiac event rate during followup [16]. Nevertheless, the cardiovascular prognosis of CAD is not only associated with endothelial function and traditional risk factors but also determined by its severity and further treatment strategies, such as optimal medication and coronary revascularization. There were few studies reporting the association between RHI and long-term cardiovascular events after DES implantation. Komura et al. found that lower RHI was correlated with angiographic in-stent restenosis (ISR) [17]. However, angiographic ISR could not represent the cardiac events completely. Therefore, we designed this prospective study to confirm the value of PAT in discriminating patients at risk of PMI as well as predicting long-term adverse cardiovascular events after DES implantation.

\section{Methods}

2.1. Ethics Statement. This study was approved by the Ethical Committee of Zhongshan Hospital, Fudan University (Approval No: B2016-018, Date: 2016/02/29). All patients provided their written, informed consent. The study was carried out in accordance with the principles of the Declaration of Helsinki.

2.2. Patient Recruitment. From May 2015 to November 2015, 268 patients suspected with stable CAD admitted for elective PCI were enrolled. Exclusion criteria were acute coronary syndrome, malignant hypertension, New York Heart Association (NYHA) class III to IV heart failure, cardiomyopathy, and cardiac valvular disease. Patients with active infection, carcinoma, immunological disorders, and liver or kidney dysfunction $\left(\mathrm{eGFR}<60 \mathrm{~mL} / \mathrm{min} / 1.73 \mathrm{~m}^{2}\right.$ or liver enzyme $>3 \times$ upper reference limit (URL)) were also excluded.

2.3. Measurement of RHI. RHI was measured with EndoPAT2000 $^{\text {тм }}$ (Itamar Medical, Israel) before coronary angiography by cardiologists who were blinded to the results of clinical characteristics and laboratory testing. This measurement was arranged in a quiet test room at 8 am-10 am with temperature set to 24-28 centigrade. All vasoactive medications (such as calcium antagonists and nitrate) were discontinued at least $24 \mathrm{~h}$ prior to testing. According to previous studies and device protocols, a blood pressure cuff was placed on one upper arm, while the other arm served as a control. PAT probes were placed on each index finger for continuous recording of the pulse signal. After a $10 \mathrm{~min}$ equilibration period, the blood pressure cuff was inflated to suprasystolic pressure $(200 \mathrm{mmHg}$ or $60 \mathrm{mmHg}$ plus systolic blood pressure) for $5 \mathrm{~min}$. Then, the cuff was deflated to induce reactive hyperemia and PAT was recorded for a further $5 \mathrm{~min}$. The pulse amplitude recordings were automatically analyzed and quantified as RHI.

\subsection{Clinical Records and Laboratory Measurements. CAD} was diagnosed when at least one lesion led to a $>50 \%$ reduction in lumen diameter in coronary angiography. Coronary angiography, syntax score evaluation, and intervention were performed by cardiologists blinded to laboratory testing and RHI. Venous serum samples were collected on admission and 16-20 hours after PCI for laboratory measurements.

2.5. Endpoints and Clinical Long-Term Follow-Up. PMI was defined as a cTnT value above the 99\% URL after the procedure. Patients were followed up with a median 18month interval through telephone consultation or outpatient clinic attendance. The primary outcomes were major adverse cardiovascular events (MACEs), including cardiac death, nonfatal myocardial infarction (MI), stroke, target vessel revascularization (TVR), and rehospitalization driven by heart failure.

2.6. Statistical Analysis. All statistical analyses were performed with SPSS for Windows, release 25.0 (IBM SPSS, Inc., Chicago, IL, USA). Continuous variables are presented as mean \pm standard deviation (SD) or median with the interquartile range (IQR). Categorical data are expressed as counts and percentages. Chi-square or Fisher's exact test was used to compare the frequency for categorical variables. Means for continuous variables were compared by Student's $\mathrm{t}$ or Mann-Whitney $\mathrm{U}$ test. Logistic regression was performed to identify risk factors and the Spearman test for correlation analyses. Kaplan-Meier survival analysis was conducted to compare the difference in incidence of MACEs. The prognostic impact of RHI was assessed with a univariable and multivariable Cox proportional hazard model, adjusted to age, male, hypertension, diabetes, smoking, low-density lipoprotein cholesterol, and hemoglobin. All $P$ values were two sided, and $P<0.05$ was considered statistically significance. Figures were plotted using GraphPad Prism, version 9.0 (GraphPad Software, San Diego, CA, USA).

\section{Results}

3.1. Study Population. 268 patients suspected with stable CAD undergoing elective coronary angiography were recruited in this study. 189 patients (70.5\%) were diagnosed 
with CAD by angiography, and 119 patients (44.4\%) accepted DES implantation. When PMI was defined as a postPCI cTnT value $>99 \%$ URL, 51 out of the 119 patients (42.9\%) had PMI.

3.2. Population Characteristics and RHI. Compared with patients without CAD, CAD patients had a lower RHI $(1.88 \pm 0.55$ vs. $2.02 \pm 0.58, P<0.05)$ (Figure 1$)$. The cohort was divided into two groups according to the median value of RHI (1.81). No difference in age, gender, blood pressure, body mass index (BMI), and history of diabetes or smoking was found (Table 1). Only triglyceride was detected higher in patients with $\mathrm{RHI} \leq 1.81(1.94 \pm 1.71$ vs. $1.59 \pm 0.95, P<0.05)$. Concomitant medications that could interfere with RHI were similar between the two groups. Nitrates, $\beta$-receptor blockades, and calcium channel blockades were withheld for at least $24 \mathrm{~h}$ before PAT measurement. Despite similarities in risk factors of $\mathrm{CAD}$, patients with $\mathrm{RHI} \leq 1.81$ had a higher syntax score $(17.3 \pm 8.3$ vs. $12.0 \pm 6.6, P<0.001)$. RHI was negatively correlated with syntax score (Spearman $r=-0.345, \quad P<0.001$ ) as well as hs-CRP (Spearman $r=-0.162, P<0.05) .60(45.8 \%)$ and $79(57.7 \%)$ patients underwent stenting in the high RHI and low RHI groups, respectively. The incidence of PMI was $37 \%$ in patients with $\mathrm{RHI}>1.81$ compared with $47.7 \%$ in patients with $\mathrm{RHI} \leq 1.81$, which, however, was not statistically significant yet.

3.3. Baseline and Procedural Characteristics of PMI. As shown in Table 2, patients with PMI had a lower RHI $(1.75 \pm 0.37$ vs. $1.95 \pm 0.50, P<0.05)$ and higher syntax score $(17.4 \pm 8.4$ vs. $13.4 \pm 6.3, P<0.01)$ compared with patients without PMI. Besides, patients with PMI tended to have higher LDL, higher total cholesterol, and higher HDL. Neither demographic characteristics, including age, gender, hypertension, diabetes, smoking, and BMI, nor clinical examinations, including creatinine, hs-CRP, lipoprotein (a), ejection fraction $(\mathrm{EF})$, and left ventricular diameter, were significantly different. Patients with PMI had more stents implanted and, therefore, longer stent length $(52.8 \pm 29.8$ vs. $38.0 \pm 21.5 \mathrm{~mm}, P<0.01)$. Antiplatelet therapy and use of statins, as well as bailout use of glycoprotein IIb\IIIa inhibitors, were similar between the two groups. No dissection, branch loss, perforation, or cardiac tamponade was witnessed (data not shown) in either group.

3.4. Risk Factors of PMI. Binary logistic analysis was performed to evaluate the role of RHI on the occurrence of PMI. Our results demonstrated that RHI (odds ratio (OR) 0.35, 95\% confidence interval $(\mathrm{CI}) 0.4-0.86, P<0.05)$ was a protective factor while stent length (OR 1.02, 95\%CI 1.01-1.04, $P<0.01$ ) was a risk factor of PMI (Table 3). Stent length could only be determined during the procedure, which made RHI a valuable indicator in the early prediction and prevention of PMI. Furthermore, we constructed a ROC curve to assess the ability of RHI to predict the occurrence of PMI. The area under the curve to predict PMI was 0.61 (95\% CI: $0.51-0.71 ; P<0.05$ ) (Figure 3). An RHI cutoff value of

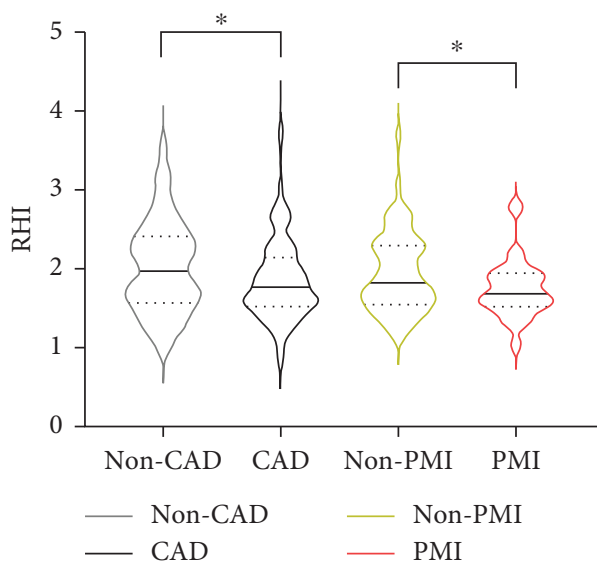

FIgURE 1: Violin plot of RHI in different groups. Distribution of RHI in different patient groups was presented in a violin plot. The width of the box represents the density of values. Solid lines and dotted lines represent the median and interquartile range of RHI, respectively. Patients with CAD or PMI had lower RHI compared with patients without $\mathrm{CAD}$ or PMI, separately. ${ }^{*} P<0.05$. CAD, coronary artery disease; PMI, periprocedural myocardial injury; and RHI, reactive hyperemia index.

1.83 had a sensitivity of $62.7 \%$ and specificity of $50.0 \%$ to detect PMI.

3.5. RHI and Long-Term Outcome. Patients were followed up for a mean period of 18 months, during which 20 patients had adverse events (Table 4). No patients died. One patient had myocardial infarction (MI). 13 patients underwent target vessel revascularization. Four patients were hospitalized for deterioration of heart failure. Ischemic stroke was diagnosed in 2 patients. Kaplan-Meier survival analysis was performed to compare the difference in the cumulative incidence of MACEs between patients with high and low levels of RHI. Using the median value of RHI (1.81), we found that patients with low RHI were more likely to suffer from MACEs (log-rank $P<0.05$ ) (Figure 2). The impact of RHI on the risk of MACEs was also evaluated by univariate and multivariate Cox regression analyses. $\mathrm{RHI} \leq 1.81$ increased the risk of MACEs in CAD patients (HR 3.34, 95\%CI $1.10-10.16 ; P<0.05)$, even after adjustment to age, gender, hypertension, diabetes, smoking, hemoglobin, and LDL (adHR 3.31, 95\%CI 1.07-10.22, $P<0.05$ ).

3.6. Discussion. In the past decades, the development of both equipment and technique has made PCI a mainstay in the treatment of obstructive coronary disease. While severe complications of PCI keep declining over the years, there has not been an ideal way to predict PMI and cut down the incidence. Coronary microvascular dysfunction plays an essential role in PMI, which is not only the consequence of microcirculatory damage but also a crucial risk factor to exacerbate myocardial injury. Assessment of CMD relies on functional assessment of microcirculation, which can be performed invasively, such as index of microvascular resistance (IMR) [18, 19], and noninvasively, including 
TABLE 1: Demographics and clinical characteristics.

\begin{tabular}{|c|c|c|c|c|}
\hline & Total (268) & RHI > $1.81(131)$ & $\mathrm{RHI} \leq 1.81(137)$ & $P$ \\
\hline Age & $62.9 \pm 9.0$ & $63.2 \pm 8.8$ & $62.6 \pm 9.3$ & 0.654 \\
\hline Male & $185(69.0)$ & $87(66.4)$ & $98(71.5)$ & 0.365 \\
\hline Hypertension & $170(63.4)$ & $80(61.1)$ & $90(65.7)$ & 0.432 \\
\hline Diabetes & $60(22.3)$ & $28(21.4)$ & $32(23.4)$ & 0.697 \\
\hline Smoking history & $95(35.4)$ & $42(32.1)$ & $53(38.7)$ & 0.257 \\
\hline BMI & $24.7 \pm 3.0$ & $24.5 \pm 3.2$ & $24.9 \pm 2.8$ & 0.228 \\
\hline $\mathrm{CAD}$ & $189(70.5)$ & $87(66.4)$ & $102(74.5)$ & 0.149 \\
\hline Syntax score & $14.9 \pm 8.0$ & $12.0 \pm 6.6$ & $17.3 \pm 8.3$ & $<0.001$ \\
\hline Coronary slow flow* & $11(4.1)$ & $4(3.1)$ & $7(5.1)$ & 0.396 \\
\hline \multicolumn{5}{|l|}{ Laboratory and auxiliary examinations } \\
\hline Systolic blood pressure (mmHg) & $132.4 \pm 12.3$ & $131.6 \pm 12.2$ & $133.2 \pm 12.4$ & 0.592 \\
\hline Diastolic blood pressure (mmHg) & $80.0 \pm 8.2$ & $79.0 \pm 8.4$ & $81.0 \pm 8.0$ & 0.100 \\
\hline NT-proBNP (pg/ml) & $214.2 \pm 536.7$ & $215.1 \pm 523.9$ & $213.0 \pm 550.6$ & 0.461 \\
\hline Creatinine $(\mathrm{mg} / \mathrm{dl})$ & $78.2 \pm 16.9$ & $76.8 \pm 17.3$ & $79.5 \pm 16.6$ & 0.187 \\
\hline $\mathrm{CK}(\mathrm{U} / \mathrm{L})$ & $94.4 \pm 47.6$ & $94.0 \pm 50.8$ & $94.8 \pm 44.4$ & 0.317 \\
\hline CK-MB (U/L) & $12.9 \pm 8.2$ & $13.0 \pm 10.2$ & $12.7 \pm 5.2$ & 0.941 \\
\hline hs-CRP (mg/L) & $3.94 \pm 7.27$ & $3.55 \pm 7.17$ & $4.35 \pm 7.40$ & 0.096 \\
\hline Hemoglobin $(\mathrm{g} / \mathrm{L})$ & $134.4 \pm 14.5$ & $132.9 \pm 13.9$ & $135.9 \pm 14.8$ & 0.091 \\
\hline Platelet $\left(\times 10^{\wedge} 9 / \mathrm{L}\right)$ & $207.5 \pm 64.2$ & $205.9 \pm 62.1$ & $209.0 \pm 66.4$ & 0.827 \\
\hline Total cholesterol $(\mathrm{mmol} / \mathrm{L})$ & $3.82 \pm 0.97$ & $3.73 \pm 0.86$ & $3.91 \pm 1.07$ & 0.252 \\
\hline Low-density lipoprotein $(\mathrm{mmol} / \mathrm{L})$ & $1.97 \pm 0.81$ & $1.94 \pm 0.74$ & $1.99 \pm 0.87$ & 0.892 \\
\hline Triglyceride $(\mathrm{mmol} / \mathrm{L})$ & $1.77 \pm 1.40$ & $1.59 \pm 0.96$ & $1.94 \pm 1.71$ & 0.038 \\
\hline High-density lipoprotein $(\mathrm{mmol} / \mathrm{L})$ & $1.14 \pm 0.46$ & $1.15 \pm 0.57$ & $1.13 \pm 0.33$ & 0.938 \\
\hline $\mathrm{Lp}(\mathrm{a})(\mathrm{mmol} / \mathrm{L})$ & $349.6 \pm 485.8$ & $351.8 \pm 468.7$ & $347.5 \pm 503.5$ & 0.370 \\
\hline $\mathrm{HbAlc}(\%)$ & $6.2 \pm 1.4$ & $6.1 \pm 1.2$ & $6.3 \pm 1.5$ & 0.111 \\
\hline \multicolumn{5}{|l|}{ Echocardiography } \\
\hline $\mathrm{LA}(\mathrm{mm})$ & $38.9 \pm 4.3$ & $38.1 \pm 4.4$ & $39.5 \pm 0.4$ & 0.010 \\
\hline LVEDD (mm) & $47.1 \pm 4.5$ & $46.5 \pm 4.2$ & $47.6 \pm 4.8$ & 0.050 \\
\hline LVESD (mm) & $30.3 \pm 4.3$ & $30.0 \pm 3.7$ & $30.8 \pm 4.8$ & 0.090 \\
\hline SPAP (mmHg) & $32.4 \pm 6.3$ & $32.7 \pm 7.0$ & $32.1 \pm 5.7$ & 0.784 \\
\hline $\mathrm{EF}(\%)$ & $64.4 \pm 7.0$ & $64.3 \pm 7.3$ & $64.5 \pm 6.6$ & 0.906 \\
\hline \multicolumn{5}{|l|}{ Medication } \\
\hline Statin & $237(88.4)$ & $113(86.3)$ & $124(90.5)$ & 0.277 \\
\hline B-blockade & $175(65.2)$ & $86(65.6)$ & $89(65.0)$ & 0.906 \\
\hline Nitrates & $132(49.3)$ & $59(45.0)$ & $73(53.3)$ & 0.177 \\
\hline Calcium channel blockade & $68(25.4)$ & $35(26.7)$ & $33(24.1)$ & 0.621 \\
\hline
\end{tabular}

Data are shown as mean $\pm \mathrm{SD}$ or $n(\%) .{ }^{*}$ Slow flow was defined as a reduced thrombolysis in a myocardial infarction (TIMI) flow grade of 2 or lower in coronary angiography. BMI, body mass index; CAD, coronary artery disease; hs-CRP, hypersensitive C-reactive protein; EF, ejection fraction; LA, left atrium; LVEDD, left ventricular end-diastolic diameter; LVESD, left ventricular end-systolic diameter; Lp (a), lipoprotein (a); PMI, periprocedural myocardial injury; SPAP, systolic pulmonary arterial pressure.

positron emission tomography (PET) and cardiac magnetic resonance (MRI) [20, 21]. However, these methods are limited by availability, cost, or exposure to radiation. RHI assessment by PAT has been validated as a noninvasive way to evaluate peripheral and coronary microvascular endothelial function $[12,14,15]$. Herein, we designed this prospective study to determine if preprocedural RHI is a risk factor and predictor of PMI. Patients with comorbidities that would influence cTnT values were excluded, such as ACS, renal disease, and autoimmune disease. In order to exclude the influence of environment factors, we conducted PAT in a solitary room at a stipulated time with constant illumination, temperature, and noise control. Medications that could interfere with PAT were withheld, and patients were inculcated to avoid stress since the night before measurement. RHI was calculated automatically by using the recorder to prevent the objective error of the operator.
In this cohort, the incidence of PMI was consistent with our previous study [22]. Compared with the patients without CAD, CAD patients had lower RHI. RHI was also negatively associated with syntax score and hs-CRP. These indicated that RHI was associated with the severity of CAD and, more importantly, the inflammatory state. This dose makes sense since previous studies have confirmed the inflammatory state is interrelated with atherosclerosis and endothelial dysfunction $[19,23,24]$. Logistic regression implied RHI was a protective factor of PMI, and ROC analysis indicated RHI could predict the occurrence of PMI with an AUC of 0.61. Among risk factors that could be identified before PCI, including hypertension, diabetes, smoking, dyslipidemia, and BMI, RHI is the only indicator that correlated with PMI. Stent length was another risk factor of PMI; however, it could not be determined before the angiography was performed, which limited its use in the early prediction of PMI. 
TABLE 2: Baseline and procedural characteristics of PMI.

\begin{tabular}{|c|c|c|c|c|}
\hline & Total (119) & Non-PMI (68) & PMI (51) & $P$ \\
\hline Age & $63.7 \pm 9.5$ & $64.0 \pm 9.8$ & $63.2 \pm 9.3$ & 0.476 \\
\hline Male & $91(76.4)$ & $50(73.5)$ & $41(80.4)$ & 0.382 \\
\hline Hypertension & $81(68.1)$ & $44(64.7)$ & $37(72.5)$ & 0.364 \\
\hline Diabetes & $25(21.0)$ & $16(23.5)$ & $9(17.5)$ & 0.687 \\
\hline Smoking history & $49(41.1)$ & $25(36.8)$ & $24(47.1)$ & 0.259 \\
\hline BMI & $24.8 \pm 3.2$ & $24.7 \pm 3.2$ & $24.9 \pm 3.3$ & 0.573 \\
\hline Syntax score & $15.2 \pm 7.5$ & $13.4 \pm 6.3$ & $17.4 \pm 8.4$ & 0.008 \\
\hline RHI & $1.86 \pm 0.46$ & $1.95 \pm 0.50$ & $1.75 \pm 0.37$ & 0.039 \\
\hline \multicolumn{5}{|l|}{ Laboratory and auxiliary examinations } \\
\hline NT-proBNP $(\mathrm{pg} / \mathrm{ml})$ & $225.1 \pm 582.9$ & $229.7 \pm 735.7$ & $219.2 \pm 298.2$ & 0.006 \\
\hline Creatinine $(\mathrm{mg} / \mathrm{dl})$ & $78.3 \pm 15.8$ & $76.6 \pm 15.0$ & $80.6 \pm 16.6$ & 0.088 \\
\hline $\mathrm{CK}(\mathrm{U} / \mathrm{L})$ & $94.6 \pm 45.6$ & $96.0 \pm 50.8$ & $92.9 \pm 38.3$ & 0.658 \\
\hline CK-MB (U/L) & $12.8 \pm 3.8$ & $12.8 \pm 4.3$ & $12.8 \pm 3.1$ & 0.596 \\
\hline hs-CRP (mg/L) & $5.07 \pm 9.41$ & $3.95 \pm 6.08$ & $6.46 \pm 12.3$ & 0.691 \\
\hline Hemoglobin $(\mathrm{g} / \mathrm{L})$ & $136.2 \pm 14.1$ & $135.4 \pm 13.8$ & $137.3 \pm 14.7$ & 0.512 \\
\hline Platelet $\left(\times 10^{\wedge} 9 / \mathrm{L}\right)$ & $208.3 \pm 68.2$ & $205.4 \pm 76.4$ & $212.3 \pm 56.0$ & 0.262 \\
\hline Total cholesterol $(\mathrm{mmol} / \mathrm{L})$ & $3.86 \pm 1.02$ & $3.70 \pm 0.99$ & $4.08 \pm 1.03$ & 0.032 \\
\hline Low-density lipoprotein $(\mathrm{mmol} / \mathrm{L})$ & $2.04 \pm 0.83$ & $1.88 \pm 0.77$ & $2.24 \pm 0.86$ & 0.024 \\
\hline Triglyceride $(\mathrm{mmol} / \mathrm{L})$ & $1.83 \pm 1.70$ & $1.97 \pm 2.10$ & $1.66 \pm 0.92$ & 0.912 \\
\hline High-density lipoprotein $(\mathrm{mmol} / \mathrm{L})$ & $1.08 \pm 0.30$ & $1.06 \pm 0.34$ & $1.12 \pm 0.24$ & 0.025 \\
\hline $\mathrm{Lp}(\mathrm{a})(\mathrm{mmol} / \mathrm{L})$ & $339.2 \pm 438.2$ & $300.0 \pm 298.6$ & $391.8 \pm 485.2$ & 0.393 \\
\hline HbAlc $(\%)$ & $6.1 \pm 1.2$ & $6.1 \pm 1.4$ & $6.1 \pm 0.9$ & 0.400 \\
\hline \multicolumn{5}{|l|}{ Echocardiography } \\
\hline $\mathrm{LA}(\mathrm{mm})$ & $39.2 \pm 4.2$ & $39.2 \pm 4.5$ & $39.4 \pm 3.8$ & 0.900 \\
\hline LVEDD (mm) & $46.7 \pm 4.0$ & $46.5 \pm 4.5$ & $47.2 \pm 3.2$ & 0.350 \\
\hline LVESD $(\mathrm{mm})$ & $30.2 \pm 3.4$ & $30.1 \pm 3.6$ & $30.4 \pm 3.1$ & 0.644 \\
\hline SPAP (mmHg) & $32.9 \pm 7.4$ & $33.1 \pm 8.2$ & $32.6 \pm 6.3$ & 0.973 \\
\hline $\mathrm{EF}(\%)$ & $64.7 \pm 6.1$ & $65.0 \pm 6.2$ & $64.5 \pm 6.2$ & 0.529 \\
\hline \multicolumn{5}{|l|}{ Medication } \\
\hline Aspirin & $115(96.6)$ & $64(94.1)$ & $51(100)$ & 0.134 \\
\hline Statin & $118(99.2)$ & $67(98.5)$ & $51(100)$ & 1.000 \\
\hline B-blockade & $87(73.1)$ & $54(79.4)$ & $34(66.7)$ & 0.117 \\
\hline Nitrates & $74(62.2)$ & $39(57.4)$ & $35(68.6)$ & 0.060 \\
\hline Calcium channel blockade & $29(24.3)$ & $16(23.5)$ & $13(25.5)$ & 0.805 \\
\hline \multicolumn{5}{|l|}{$P C I$} \\
\hline Stent length (mm) & $44.4 \pm 26.3$ & $38.0 \pm 21.5$ & $52.8 \pm 29.8$ & 0.002 \\
\hline Use of GBI & $20(16.8)$ & $11(16.2)$ & $9(17.6)$ & 0.832 \\
\hline Average stent diameter $(\mathrm{mm})$ & $3.06 \pm 0.44$ & $3.11 \pm 0.45$ & $2.99 \pm 0.41$ & 0.149 \\
\hline \multicolumn{5}{|l|}{ Target vessel number } \\
\hline 1 & $96(80.7)$ & $58(85.3)$ & $38(74.5)$ & 0.140 \\
\hline 2 & $20(16.8)$ & $9(13.2)$ & $11(21.6)$ & 0.229 \\
\hline 3 & $3(2.5)$ & $1(1.5)$ & $2(1.7)$ & 0.576 \\
\hline
\end{tabular}

Data are shown as mean \pm SD or $n$ (\%). *Slow flow was defined as a reduced thrombolysis in a myocardial infarction (TIMI) flow grade of 2 or lower in coronary angiography. BMI, body mass index; hs-CRP, hypersensitive C-reactive protein; GPI, glycoprotein IIb/IIIa receptor inhibitor; EF, ejection fraction; LA, left atrium; LVEDD, left ventricular end-diastolic diameter; LVESD, left ventricular end-systolic diameter; Lp (a), lipoprotein (a); PCI, percutaneous coronary intervention; SPAP, systolic pulmonary arterial pressure.

Although the sensitivity and specificity of RHI require validation in larger populations, PAT proves to be a promising noninvasive method to predict PMI early and conveniently. The median value of RHI was 1.81 in our study, which was different from previous reports $[25,26]$. Variance in the level of RHI may be associated with different inclusion criteria, races, age distributions, and diseases of interest. Larger-scale studies are needed to determine a uniform cutoff value of RHI for risk identification.

Moreover, RHI is reported to be closely related with risk factors and early stage of CAD as well as poor cardiovascular prognosis $[13,14,16,27]$. In this study, we did not find the association between RHI and traditional risk factors, such as diabetes, hypertension, and BMI. Probably, it is because the sample size was relatively small. Besides, these patients recruited had less comorbidities and relatively short course of disease, in which case the influence of risk factors has not posed an impact strong enough on RHI. DES has become one of the most important treatments in CAD; nevertheless, few studies reported the impact of RHI on long-term outcome after DES implantation. We found that the median level of RHI (1.81) was associated with a higher incidence of MACEs, even adjusted by traditional risk factors. It has been argued that PMI as well as PMI-related poor prognosis could 
TABLE 3: Risk factors of PMI.

\begin{tabular}{lcr}
\hline & Odds ratio $(95 \%$ CI $)$ & $P$ \\
\hline Age $\geq 65$ y & $0.67(0.33-1.40)$ & 0.291 \\
Male & $1.48(0.61-3.55)$ & 0.384 \\
Hypertension & $1.44(0.65-3.18)$ & 0.365 \\
Diabetes & $0.70(0.28-1.73)$ & 0.437 \\
Dyslipidemia & $1.47(0.64-3.38)$ & 0.362 \\
Smoking history & $1.53(0.73-3.20)$ & 0.260 \\
Body mass index & $1.02(0.91-1.14)$ & 0.738 \\
Stent length & $1.02(1.01-1.04)$ & 0.004 \\
Target vessel number & $1.82(0.83-4.00)$ & 0.137 \\
Average stent diameter & $0.52(0.22-1.24)$ & 0.141 \\
RHI & $0.35(0.14-0.86)$ & 0.022 \\
\hline
\end{tabular}

RHI, reactive hyperemia index.

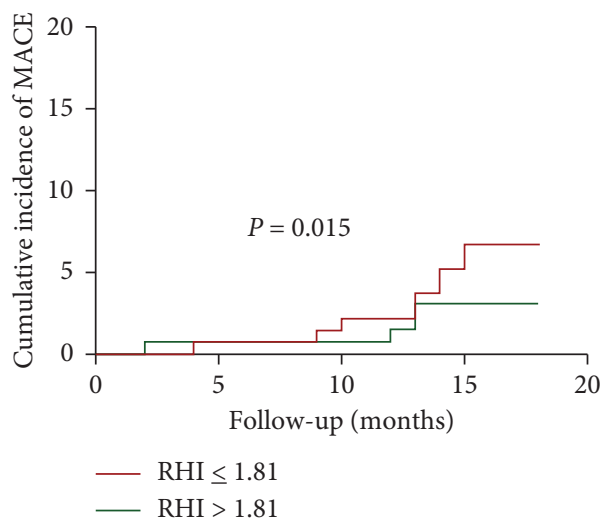

Figure 2: Cumulative incidence of MACEs. Patients with RHI $\leq 1.81$ had a higher risk of MACEs $(P<0.05)$. MACEs, major adverse cardiovascular events; RHI, reactive hyperemia index.

TABLE 4: Long-term outcomes and RHI.

\begin{tabular}{lccccc}
\hline & RHI $\leq 1.81$ & RHI $>1.81$ & HR & $P$ & adHR \\
\hline MACEs* & 16 & 4 & $3.34(1.10-10.16)$ & 0.033 & $3.31(1.07-10.22)$ \\
Nonfatal myocardial infarction & 1 & 0 & - & 0.038 \\
Target vessel revascularization & 10 & 3 & - & 0.193 & - \\
Cardiac death & 0 & 0 & - & - & - \\
Rehospitalization driven by heart failure & 4 & 0 & - & 0.784 & - \\
Ischemic stroke & 1 & 1 & - & 0.053 & - \\
\hline
\end{tabular}

Values are given as $n$. Adjustments were made for age $(>65$ y), gender, hypertension, diabetes, smoking, hemoglobin, and LDL. Only data with $P<0.05$ were shown. ${ }^{*}$ MACEs were a composite of cardiac death, nonfatal myocardial infarction, ischemic stroke, target vessel revascularization, and rehospitalization driven by heart failure.

be a result of plaque burden or procedural complexity. In the present study, we also noticed RHI was adversely associated with syntax score, which reflected the status of atherosclerosis. However, as we mentioned in the previous paper [22], PMI posed a greater impact on patients with low risk of adverse cardiovascular events, which could not be explained by either coronary lesions or the procedure. Therefore, endothelial and microcirculatory dysfunction could be a crucial cause of poor prognosis, which was not included in syntax score but an appropriate target of PAT. Besides, we have built multivariate predictive models of MACEs based on traditional risk factors with and without RHI. Preliminary results indicated inclusion of PAT improved C-statistic and decreased the Akaike information criterion even in the presence of syntax score; that is, RHI proved to be an important predictor of MACEs holding additional value. Due to the relatively small number of patients, these results were tentative and not displayed yet. Moreover, PAT is a convenient method to be conducted, which can be a valuable way to track the fluctuation of RHI in order to assess the effectiveness of therapy and guiding clinical practice in the future.

There are some limitations in our study. First, this is a single-centered study with a relatively small sample size. However, the present study has been a relatively large one to investigate the independent role of RHI on the long-term 


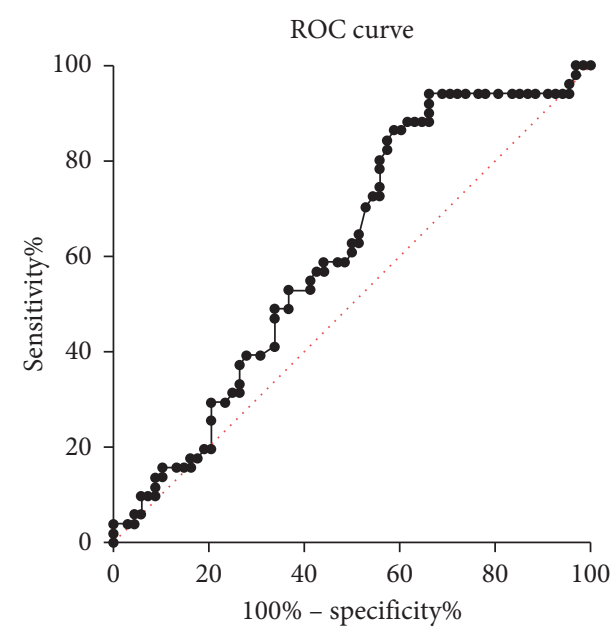

FIgure 3: ROC curve for the prediction of PMI. The dotted diagonal line is the null hypothesis with $\mathrm{AUC}=0.50 \mathrm{RHI}<1.83 \mathrm{had}$ a sensitivity of $62.7 \%$ and specificity of $50.0 \%$ to predict PMI with $\mathrm{AUC}=0.61$ (95\% CI 0.51-0.71). AUC, area under the curve; CI, confidence interval; PMI, periprocedural myocardial injury; RHI, reactive hyperemia index; ROC, receiver operating characteristic.

composite outcome after DES implantation. Second, this study was conducted on a rather homogenous population. Patients with comorbidities such as ACS and renal or liver dysfunction were excluded. As a result, additional research is needed to verify if these findings could be extended to other cohorts. Third, we could not acquire the details about RHI when MACEs happened, for RHI measured at follow-up may reflect the simultaneous state of endothelial function and more closely related with the type of MACEs. However, this will not affect the conclusion that baseline RHI was a predictor of PMI and correlated with high risk of MACEs. Fourth, multicentered research is pended to bring out a uniform cutoff value of RHI to predict, and the specificity and sensitivity of RHI need to be tested in larger populations.

\section{Conclusions}

RHI assessed by PAT could be a promising predictor of PMI before the procedure. Low RHI is correlated with high risk of long-term MACEs in CAD patients after DES implantation.

\section{Data Availability}

Readers can access the data supporting the conclusions of this study by contacting the correspondence author.

\section{Conflicts of Interest}

There are no conflicts of interest pertaining to this submission.

\section{Authors' Contributions}

Zhangwei Chen, You Zhou, and Jiasheng Yin contributed equally to this article.

\section{Acknowledgments}

This study was supported by the National Program on Key Basic Research Project of China (Grant Nos. 2019YFC0840601 and 2014CBA02003), National Natural Science Foundation of China (Grant Nos. 81870267, 81970295, 81521001, 81670318, and 81570314), grant of Shanghai Shenkang on Key Clinical Research Project (Grant Nos. SHDC2020CR2015A and SHDC12019104), grant of Shanghai Science and Technology Committee (Grant Nos. 19MC1910300, 18411950200, and 20JC1410800), Key Medical and Health Projects of Xiamen Province (No. 3502Z20204004), grant of Shanghai Municipal Commission of Health and Family Planning (Grant No. 2017YQ057), grant of Zhongshan Hospital Affiliated to Fudan University (Grant No. 2018ZSLC01), VG Funding of Clinical Trials (2017-CCA-VG-036) and Merck Funding (Xinxin-merckfund-051), and National Key Research and Development Program of China from the Ministry of Science and Technology of the People's Republic of China (2016YFC1301203).

\section{References}

[1] C. W. Lee, J.-M. Ahn, R. Cavalcante et al., "Coronary artery bypass surgery versus drug-eluting stent implantation for left main or multivessel coronary artery disease," JACC: Cardiovascular Interventions, vol. 9, no. 24, pp. 2481-2489, 2016.

[2] G. Ganesha Babu, J. Malcolm Walker, D. M. Yellon, and D. J. Hausenloy, "Peri-procedural myocardial injury during percutaneous coronary intervention: an important target for cardioprotection," European Heart Journal, vol. 32, no. 1, pp. 23-31, 2011.

[3] H. M. Garcia-Garcia, E. P. McFadden, C. von Birgelen et al., "Impact of periprocedural myocardial biomarker elevation on mortality following elective percutaneous coronary intervention," JACC: Cardiovascular Interventions, vol. 12, no. 19, pp. 1954-1962, 2019.

[4] D.-W. Park, Y.-H. Kim, S.-C. Yun et al., "Frequency, causes, predictors, and clinical significance of peri-procedural myocardial infarction following percutaneous coronary intervention," European Heart Journal, vol. 34, no. 22, pp. 1662-1669, 2013.

[5] M. Zeitouni, J. Silvain, P. Guedeney et al., "Periprocedural myocardial infarction and injury in elective coronary stenting," European Heart Journal, vol. 39, no. 13, pp. 1100-1109, 2018.

[6] H. Idris, S. Lo, I. M. Shugman et al., "Varying definitions for periprocedural myocardial infarction alter event rates and prognostic implications," Journal of the American Heart Association, vol. 3, Article ID e001086, 2014.

[7] J. Herrmann, "Peri-procedural myocardial injury: 2005 update," European Heart Journal, vol. 26, no. 23, pp. 2493-2519, 2005.

[8] D. Bonderman, A. Teml, J. Jakowitsch et al., "Coronary noreflow is caused by shedding of active tissue factor from dissected atherosclerotic plaque," Blood, vol. 99, no. 8, pp. 2794-2800, 2002.

[9] N. Saleh, B. Svane, J. Jensen, L.-O. Hansson, M. Nordin, and P. Tornvall, "Stent implantation, but not pathogen burden, is associated with plasma C-reactive protein and interleukin-6 levels after percutaneous coronary intervention in patients 
with stable angina pectoris," American Heart Journal, vol. 149, no. 5, pp. 876-882, 2005.

[10] L. Gregorini, J. Marco, B. Farah et al., "Effects of selective $\alpha_{1}$ -and $\alpha_{2}$-adrenergic blockade on coronary flow reserve after coronary stenting," Circulation, vol. 106, no. 23, pp. 2901-2907, 2002.

[11] G. Heusch, A. Skyschally, and P. Kleinbongard, "Coronary microembolization and microvascular dysfunction," International Journal of Cardiology, vol. 258, pp. 17-23, 2018.

[12] E. Vizzardi, M. Gavazzoni, P. Della Pina et al., "Noninvasive assessment of endothelial function," Journal of Investigative Medicine, vol. 62, no. 6, pp. 856-864, 2014.

[13] N. M. Hamburg, M. J. Keyes, M. G. Larson et al., "Crosssectional relations of digital vascular function to cardiovascular risk factors in the Framingham heart study," Circulation, vol. 117, no. 19, pp. 2467-2474, 2008.

[14] P. O. Bonetti, G. M. Pumper, S. T. Higano, D. R. Holmes, J. T. Kuvin, and A. Lerman, "Noninvasive identification of patients with early coronary atherosclerosis by assessment of digital reactive hyperemia," Journal of the American College of Cardiology, vol. 44, no. 11, pp. 2137-2141, 2004.

[15] M. Nardone, S. Miner, M. McCarthy, C. I. Ardern, and H. Edgell, "Noninvasive microvascular indices reveal peripheral vascular abnormalities in patients with suspected coronary microvascular dysfunction," Canadian Journal of Cardiology, vol. 36, no. 8, pp. 1289-1297, 2020.

[16] R. Rubinshtein, J. T. Kuvin, M. Soffler et al., "Assessment of endothelial function by non-invasive peripheral arterial tonometry predicts late cardiovascular adverse events," European Heart Journal, vol. 31, no. 9, pp. 1142-1148, 2010.

[17] N. Komura, K. Tsujita, K. Yamanaga et al., "Impaired peripheral endothelial function assessed by digital reactive hyperemia peripheral arterial tonometry and risk of In-stent restenosis," Journal of the American Heart Association, vol. 5, no. 6, Article ID e003202, 2016.

[18] A. Suda, J. Takahashi, K. Hao et al., "Coronary functional abnormalities in patients with angina and nonobstructive coronary artery disease," Journal of the American College of Cardiology, vol. 74, no. 19, pp. 2350-2360, 2019.

[19] A. Nakayama, J. Albarrán-Juárez, G. Liang et al., "Disturbed flow-induced Gs-mediated signaling protects against endothelial inflammation and atherosclerosis," JCI Insight, vol. 5, no. 23, Article ID e140485, 2020.

[20] F. Vancheri, G. Longo, S. Vancheri, and M. Henein, "Coronary microvascular dysfunction," Journal of Clinical Medicine, vol. 9, no. 9, p. 2880, 2020.

[21] H. Rahman, C. M. Scannell, O. M. Demir et al., "High-resolution cardiac magnetic resonance imaging techniques for the identification of coronary microvascular dysfunction," JACC Cardiovasc Imaging, vol. 14, no. 5, pp. 978-986, 2020.

[22] Y. Zhou, Z. Chen, J. Ma et al., "Incidence, predictors and clinical significance of periprocedural myocardial injury in patients undergoing elective percutaneous coronary intervention," Journal of Cardiology, vol. 76, no. 3, pp. 309-316, 2020.

[23] W. J. Paulus and C. Tschöpe, "A novel paradigm for heart failure with preserved ejection fraction," Journal of the American College of Cardiology, vol. 62, no. 4, pp. 263-271, 2013.

[24] G. Grandl and C. Wolfrum, "Hemostasis, endothelial stress, inflammation, and the metabolic syndrome," Seminars in Immunopathology, vol. 40, no. 2, pp. 215-224, 2018.

[25] A. Suessenbacher, J. Dörler, M. Wanitschek, H. F. Alber, O. Pachinger, and M. Frick, "Prognostic value of peripheral arterial tonometry in patients with coronary artery disease and a high cardiovascular risk profile," Journal of Atherosclerosis and Thrombosis, vol. 21, no. 3, pp. 230-238, 2014.

[26] A. W. Schoenenberger, N. Urbanek, M. Bergner, S. Toggweiler, T. J. Resink, and P. Erne, "Associations of reactive hyperemia index and intravascular ultrasoundassessed coronary plaque morphology in patients with coronary artery disease," The American Journal of Cardiology, vol. 109, no. 12, pp. 1711-1716, 2012.

[27] K. Fujisue, S. Sugiyama, T. Ono et al., "Effects of endothelial dysfunction on residual platelet aggregability after dual antiplatelet therapy with aspirin and clopidogrel in patients with stable coronary artery disease," Circulation: Cardiovascular Interventions, vol. 6, no. 4, pp. 452-459, 2013. 